\title{
Development of a High-Level Simulation Approach and Its Application to Multicore Video Decoding
}

\author{
Florian H. Seitner, Member, IEEE, Michael Bleyer, Member, IEEE, Margrit Gelautz, Senior Member, IEEE, \\ and Ralf M. Beuschel
}

\begin{abstract}
In this paper, we introduce a high-level simulation methodology for the modeling of multicore video processing architectures. This method allows design space explorations of parallel video processing applications (VPAs). It is used to test the performance of running a VPA on arbitrary virtual hardware and software configurations. The method represents an alternative to performing a "complete" decoder implementation on a field-programmable gate array or an applicationspecific integrated circuit. The use of our method, therefore, yields the advantage of being considerably more time, labor, and cost efficient. As an application, we use our method for designing a parallel $\mathbf{H . 2 6 4}$ decoder targeting $720 \mathrm{p} 25$ resolution at bit-rates up to $50 \mathrm{Mb} / \mathrm{s}$. Starting from a single-core decoder implementation, we use our simulator for estimating the performance gain when using a multicore architecture. We then detect the major performance bottlenecks in this multicore system and perform additional decoder splittings accordingly until we reach the targeted requirements. The use of functional splitting (i.e., pipelining) and data-parallel processing is demonstrated. The final H.264 decoder architecture is capable of fulfilling our performance requirements.
\end{abstract}

Index Terms-Design exploration, H.264 decoder, parallel architectures, video processing.

\section{INTRODUCTION}

I $\mathrm{N}$ RECENT years, a wide range of advanced video algorithms in areas such as video coding have been introduced. However, the computational complexity of these sophisticated algorithms often poses serious challenges on current processor architectures.

For reaching the computational requirements of applications such as H.264 decoding, platforms with multiple cores have emerged. These platforms distribute the application onto multiple processing units. The splitting of an application is

Manuscript received January 1, 2009; revised May 24, 2009 and July 31, 2009. First version published September 1, 2009; current version published October 30, 2009. This work was supported by the Austrian Federal Ministry of Transport, Innovation, and Technology, under the Forschung, Innovation, Technologie für Informations technologien project Video Engine Design Methodology Rules, 812429. The work of M. Bleyer was supported by the Austrian Science Fund (FWF) under Project P19797. This paper was recommended by Associate Editor E. S. Jang.

F. H. Seitner, M. Bleyer, and M. Gelautz are with Vienna University of Technology, A-1040 Vienna, Austria (e-mail: seitner@ims.tuwien.ac.at; bleyer@ims.tuwien.ac.at; gelautz@ims.tuwien.ac.at).

R. M. Beuschel is with DSP-Weuffen, Amtzell D-88279, Germany (e-mail: schreier@ims.tuwien.ac.at). The work described in this paper was carried out under his previous employment at Vienna University of Technology.

Color versions of one or more of the figures in this paper are available online at http://ieeexplore.ieee.org.

Digital Object Identifier 10.1109/TCSVT.2009.2031523 accomplished by using functional pipelining and data-parallel splitting [1].

In this paper, we propose a methodology for simulating the runtime behavior of video processing applications (VPAs) running on a multicore system. Our simulator is intended to be used at an early stage in the development process of multicore video processing architectures. In this development phase, it is crucial to have means for testing virtual hard and software configurations. One concrete example presented in this paper is given as follows. The system designer is given the task of developing an high-definition (HD) H.264 video decoder. The designer might have an idea about the multicore platform that will come to use as well as about the software partitioning into this multicore system. The proposed simulator can then be applied to test whether the resulting hard and software configuration is capable of decoding an HD stream in real-time. If this is not the case, the designer can exploit our simulator to evaluate alternative software splittings or hardware components with relatively little effort, until the performance results are convincing. We, therefore, see our simulator as an important tool for avoiding wrong decision in the early phase of system development. It is typically very time and cost-intensive to repair such wrong decisions at later stages, when such mistakes become visible (e.g., the hardware is notoriously under proportioned).

Estimating the runtime behavior of a parallel VPA is a nontrivial task due to the following two reasons. Firstly, for estimating the concurrent behavior of a multicore video processing system, one has to consider data-dependencies between the processing units appropriately. Complex VPAs such as the H.264 standard impose a large number of such data-dependencies at every granularity level of the application (e.g., in the case of H.264, the pixel, syntax, macroblock, slice, and frame-levels). Secondly, the runtime of many video algorithms depends on the video input signal (e.g., contentadaptive processing for filtering, change detection, motion estimation, and entropy coding). Each video processing step requires different runtimes for processing the individual image regions. Depending on the video stream that is given as an input, strong variations in the workload can occur. In a multicore system, the impact of workload variations at each core and the algorithmic dependencies resulting from the stream content will strongly influence the behavior of our video application.

We believe that the proposed method for estimating the computational complexity is preferable over existing 
high-level techniques, since existing techniques are typically not suited for the complex nature of multicore systems. They can, in general, not consider the concurrency and interprocessor dependencies inherent to multicore systems. A valid alternative to our method is represented by hardware-related design approaches such as hardware (HW)/software (SW) codesign methods. These methods can handle concurrency and inter-processor dependencies. However, for simulating the runtime behavior of a parallel VPA, these approaches require a fully integrated architecture (i.e., specification of the interfaces between the components) and a partitioned software. Due to the vast amount of work that is required to implement this approach, early complexity estimations are hardly possible and the flexibility to explore different parallel software designs is limited.

We summarize the contributions of this paper as follows. We propose a new methodology for the design of parallel video processing architectures. Our methodology avoids the shortcomings of related estimation techniques. The method enables addressing the runtime behavior of a VPA with multiple cores appropriately and at an early stage of the architecture's design process. Our method applies the results of existing single-core performance estimation techniques and exploits them to simulate the behavior of multicore environments. We propose a simulator based on this methodology, namely the partition assessment simulator (PAS). We verify its accuracy on a hardware-proven architecture. As an application example, we apply the PAS for designing a multicore H.264 decoder for 720 p25 streams that can handle bit-rates up to $50 \mathrm{Mb} / \mathrm{s}$.

The remainder of this paper is structured as follows. In Section II, we provide a systematic overview of existing complexity estimation techniques for video processing platforms. We analyze their shortcomings when it comes to multicore architecture design. Section III uses an H.264 decoder to demonstrate the runtime variations and dependencies typically occurring in complex VPAs. The section investigates the profiling information one obtains from common singlecore VPA implementations. We then introduce our design methodology and the multicore simulator (referred to as the partition assessment simulator) in Section IV. The design of an HD multicore H.264 decoder is described in Section V. Finally, our conclusion is given in Section VI.

\section{RELATED WORK}

When designing a multicore video processing platform, it is difficult to know what hardware will be required for a certain video application and what partitioning can exploit the available resources most efficiently. For estimating the architecture behavior, various analysis techniques have evolved. In this section, we will describe them in more detail.

\section{A. Static Analysis}

Estimating the runtime of a program is addressed by the computational complexity theory [2]. Static algorithm analysis [3] and worst case execution time [4] estimation represent more advanced methods. These techniques analyze an algorithm's definition (e.g., its source code) for estimating the com- putational complexity of a system. Path analysis for estimating upper and lower runtime bounds has been described in [5]. These methods are of limited attractiveness for our purpose, since they cannot easily be bound to a specific application and platform. In addition, they do, in general, not consider the influence of the input data on the program's execution behavior (e.g., input-dependent recursions and branches). Therefore, they only allow limited precision in predicting a program's runtime complexity.

\section{B. Profiler-Based Analysis}

For considering the impact of the input data on the program execution, dynamic profiling methods [6], [7] have been developed. These methods either simulate or extend the program in a way that the program's execution behavior can be observed during runtime. This allows measuring the complexity of decoding functions in the context of a specific input stream. The profiling procedure typically outputs measurements of consumed time or cycle counts of the investigated program running on a specific hardware [8], [9]. In this paper, we will make use of such profiling information. Single-processor profiling information is taken as an input for our simulator to estimate the behavior of a virtual multicore system.

\section{Architecture-Independent Dynamic Analysis}

In order to predict an algorithm's complexity independently from the underlying processor or compiler optimizations, architecture-independent approaches have evolved. Ravasi and Mattavelli [10] have introduced the software instrumentation tool. Traditional $\mathrm{C}$ source code is extended to instrumented $\mathrm{C}++$ classes. In contrast to simple dynamic complexity profiles that only count runtime cycles, the tool allows counting how often individual arithmetic and memory load/store operations are executed during program execution. It is important to note that these counts are architecture independent. Knowing the specific properties of a processor type (e.g., number of arithmetic units, instruction set, and register size), predictions about the runtime complexity of a decoder on the virtual hardware can be made.

In a similar spirit, the ATOMIUM tool [11] performs highlevel transformation of $\mathrm{C}$ code. The focus of this tool lies on memory analysis. A data transfer and storage exploration methodology is provided. Using this methodology, C code can be optimized in terms of execution time, memory size, and power consumption.

The profiler-based techniques described above have proven to be useful for measuring the complexity requirements of single-core video decoding systems. However, making predictions for systems with multiple cores based on these results is not straightforward. Algorithmic dependencies, differences in workload and resource limitations result in varying runtime constraints between the processing units. For video decoding systems with strong variations in the runtime complexity, making predictions about the parallel decoding system's behavior is hardly possible. Apart from the method presented in this paper, hardware-based approaches can also overcome this problem. These hardware-based methods are discussed in the following. 


\section{Hardware-Based Design Analysis}

Approaches based on synthesis and simulation of hardware description languages (HDL) [12] are able to simulate the behavior of complex architectures. They require a detailed and well-specified system architecture for performing simulations. The hardware components and interfaces as well as the partitioned decoder software must be known completely.

HW/SW-codesign methods [13], [14] provide a fluid transition from software to hardware implementation. They focus on interface design and system verification. Analogously to hardware-simulation approaches, they require a completely integrated system. A decision about the decoder splitting is already necessary at a stage where the workload distribution and the core inter-dependency of our system are not known exactly.

Hardware-based approaches are typically time and laborintensive, since the system has to be fully functional before its runtime behavior can be simulated. This limits the flexibility for experimenting with different multicore architecture configurations.

\section{E. Comparison to Our Approach}

As opposed to previous work, the proposed PAS focuses on the runtime estimation of complex multicore architectures running video processing algorithms. It is capable of predicting the application's runtime based on information gained from traditional profilers. The advantage is that our approach is already applicable at an early stage of the architecture development. In contrast to hardware-based development techniques, the labor intensive task of adapting the hardware as well as the algorithm's software is avoided. This results in a highflexibility when exploring different hardware configurations and software partitionings. One can, therefore, compare different competing software and architecture configurations against each other to find the best-suited one. According to the achieved results, the actual hardware compilation and software implementation can be performed with considerably reduced risk of being trapped in an early wrong decision.

\section{AnAlysis of ThE Single-CoRE H.264 DeCODER IMPLEMENTATION}

We use the H.264 video standard for demonstrating the challenges of parallel VPA architecture design. We have chosen an H.264 decoder for various reasons. Firstly, for achieving a high-compression efficiency, advanced coding tools such as improved spatial and temporal prediction (i.e., motion prediction up to quarter-pel accuracy), context-adaptive entropy coding and advanced deblocking filters have been introduced in this standard. Most of these tools strongly adapt to the video content and come at the cost of a highcomputational complexity and variations in the runtime of the decoder. Secondly, the H.264 algorithm contains a large number of data-dependencies at various levels of detail. For an efficient parallelization, these dependencies must be resolved appropriately.

Fig. 1 visualizes the H.264 decoding process at macroblock level. Macroblocks (MBs) form the basic processing units in block-based video coding systems. As shown in Fig. 1, the first step in decoding an MB is bit-stream parsing. The MB's syntax elements are then entropy decoded and prepared for the subsequent decoding tasks. These tasks include the spatial and temporal prediction of the video content, the inverse transformation and quantization (IDCT) of the residual information (i.e., the difference between prediction and original content) and the deblocking for removing blocking artifacts introduced by the quantization.

In the following, we analyze the runtime behavior of an existing H.264 decoder running on a single-core. This analysis builds the foundation for the development of our multicore simulation approach.

\section{A. Runtime Profiling}

Traditional dynamic profiling provides runtime information for each decoding function. In our implementation, such profiles are derived from a single-core instruction-set (IS) simulator for the multimedia processor CHILI [15]. This reduced instruction set computer processor can process four instructions in parallel which can be any combination of 32-bit arithmetic instructions and load-store operations. For parallel pixel operations, 16-bit single instruction multiple data (SIMD) instructions are provided. Each processor has a dedicated $64 \mathrm{kB}$ local memory for fast data access and a $64 \mathrm{kB}$ instruction cache. Data are transferred via direct memory access (DMA) or direct access between the processor's local memory and $64 \mathrm{kB}$ shared on-chip static random access memory (SRAM) as well as up to $64 \mathrm{MB}$ external memory.

We have extended our IS simulator for the CHILI processor and its data memory system to provide the time of each function call and each function return that has occurred during the execution of the decoder (Fig. 2). Note that in this paper we have used the CHILI profiler for obtaining this information. Nevertheless, this is no restriction of our approach, since profilers for other platforms are, in general, also able to provide this information.

Using the profiling information of each function call, we can compute the percentage of the total runtime spent in the individual decoding functions and the runtime variations occurring during the decoding process.

However, making assumptions about a parallel decoder based on these functional profilings is not straightforward. Representing the runtime information at the same processing level where the parallelization of the VPA effectively takes place is more suitable for multicore runtime predictions.

\section{B. Mapping to Parallelization Level}

Using the time of function calls and returns, we can determine the computation time that an individual MB spends in the decoding steps depicted in Fig. 1. For example, we want to know the computation time that is required for the entropy decoding of the third MB in the stream. We know that two different functions $f_{1}$ and $f_{2}$ implement entropy decoding. Let us assume that each of these functions is called exactly once in the decoding of a MB. The sum of processing times for the third invocation of $f_{1}$ and the third invocation of $f_{2}$ will 


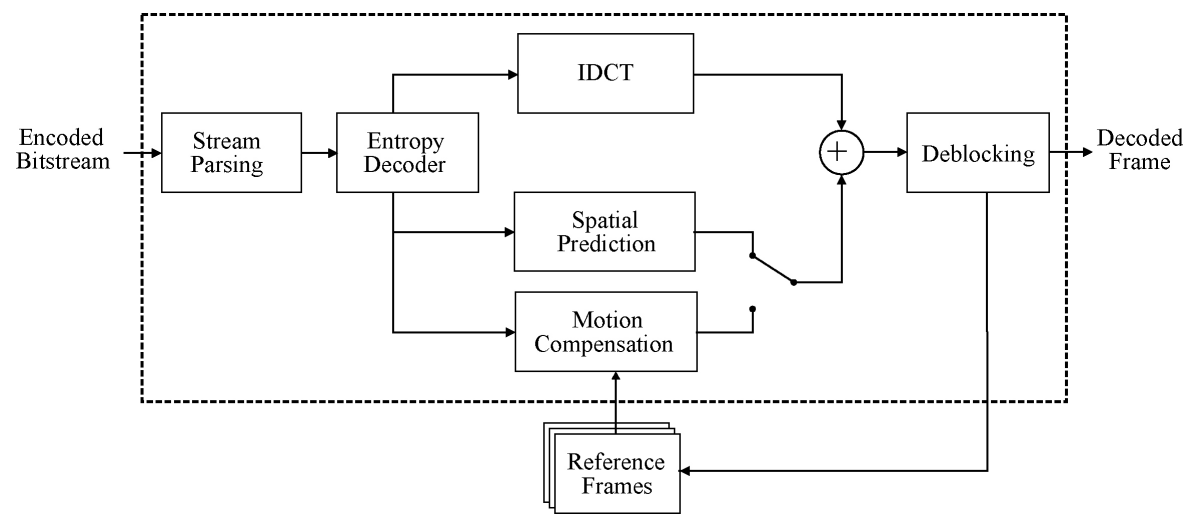

Fig. 1. H.264 decoding process. Macroblocks run through the decoder pipeline, one after the other.

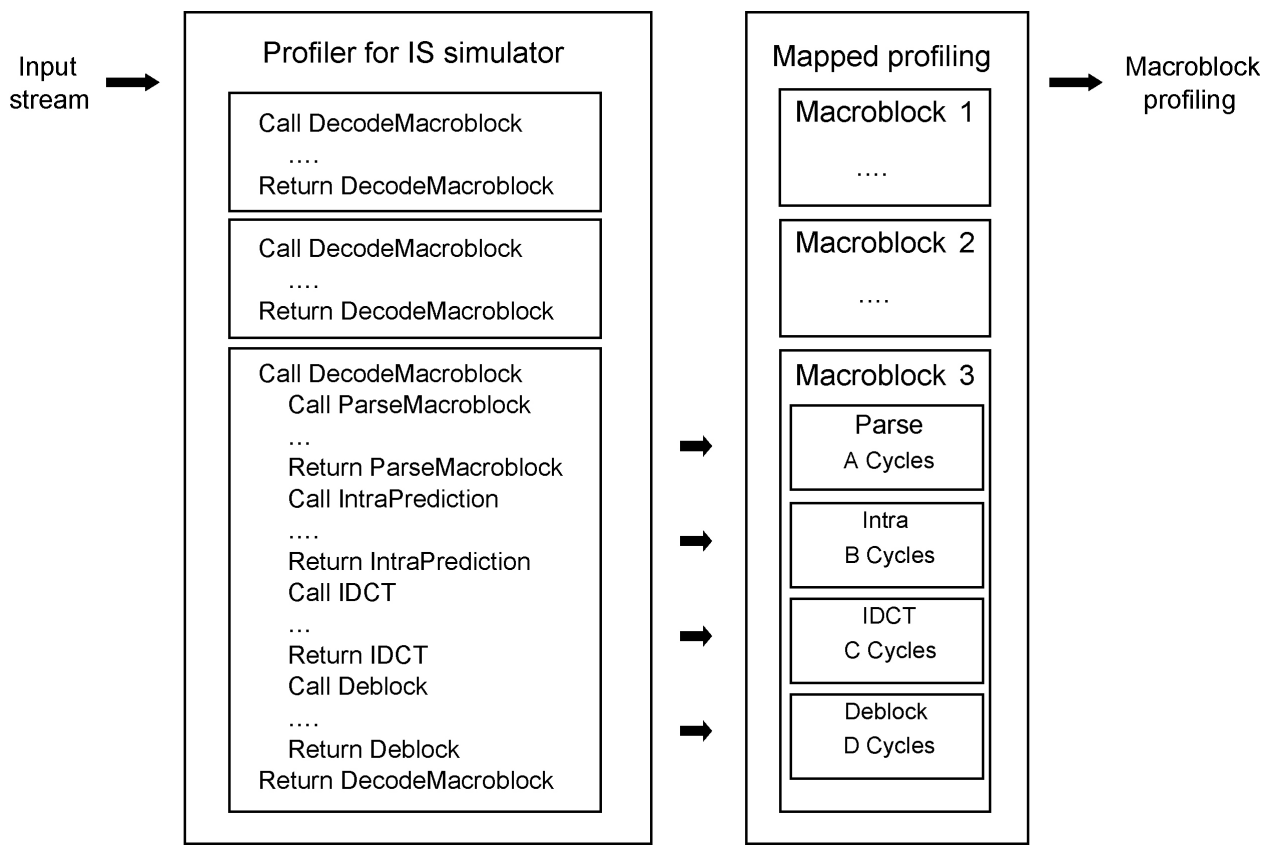

Fig. 2. Dynamic profiler delivers the exact execution times for function calls and returns. We map this information to the major decoding functions of each macroblock and calculate the number of cycles spent in each decoding block. We refer to this macroblock-assigned profiling information as macroblock profiling.

TABLE I

Four Test Sequences at 720 p Resolution. The Bottom Row Shows the Resulting Bit-Rates [At 25 Frames Per SECOnd (Frames/S)] AND THE FRAME RESOLUTION IN PIXELS

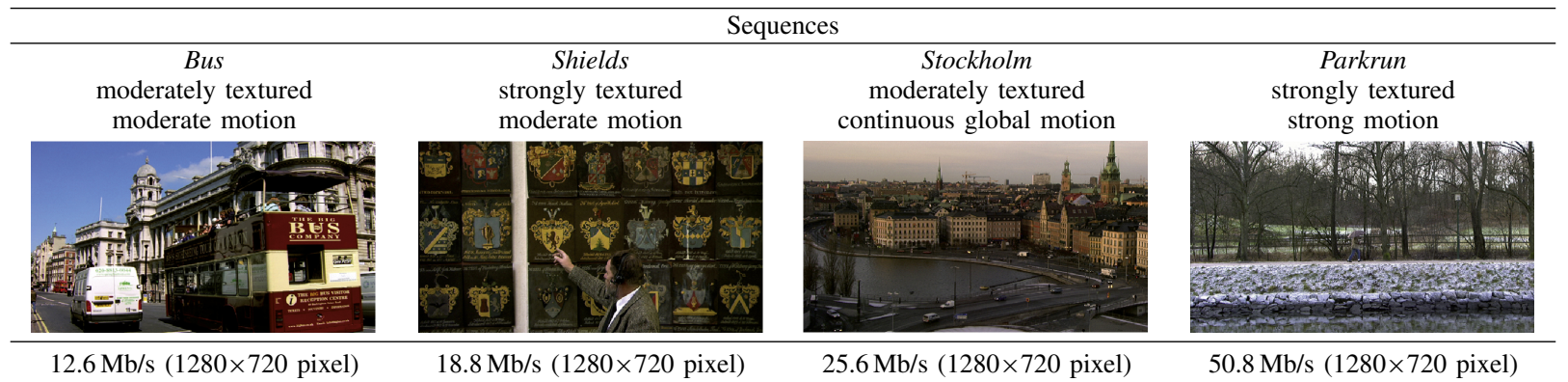



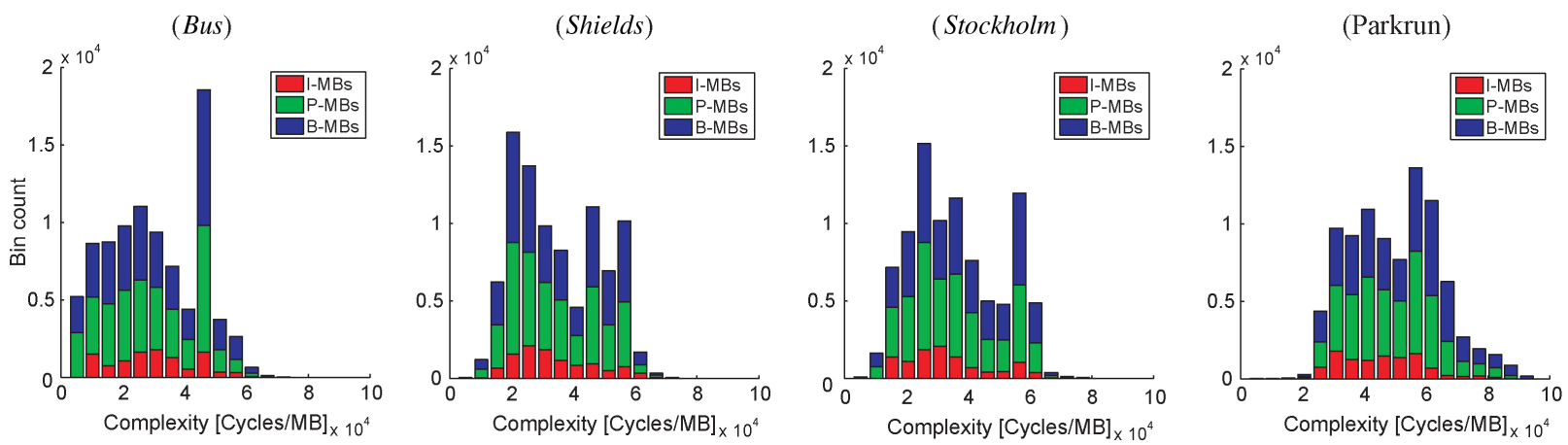

Fig. 3. Dynamic variations in the execution times of individual macroblocks in the H.264 decoding process. Histogram bins plot the number of macroblocks having similar runtimes. The colors indicate the contributions of macroblocks from I, P, and B-slices to the overall bin counts. Histograms are shown for 25 frames of the four sequences in Table I. These sequences are coded in an IPB picture coding order and a group of pictures size 11. It is observed that the runtimes of macroblocks vary considerably within a sequence. This observation is also made when considering I, P, and B-macroblocks separately.

consequently represent the time that MB 3 spends for entropy decoding. Fig. 2 visualizes this idea. However, notice that this mapping is considerably more complex in practice, since functions are typically called arbitrary times for the decoding of a single MB, which depends on the MB's coding mode. We have, therefore, implemented a state machine that performs the mapping of raw profiling data to meaningful function units. This state machine extracts the duration and the type (e.g., "ENTROPY," "INTRA," "DEBLOCK") of each MB's decoding task.

\section{Decoder Software}

The profiles are based on a commercial H.264/AVC main profile decoder for embedded architectures. This decoder has been optimized in terms of memory usage and support of DMA transfers. In addition, the regular pixel-based processing functions of the decoder (e.g., interpolation, prediction) have been assembly optimized to make use of the SIMD processor commands.

\section{Test Sequences}

We use four HD test sequences that are shown in Table III-A. At a peak signal-to-noise ratio of approximately $40 \mathrm{db}$, the four test sequences have bit-rates between 12.6 and $50.8 \mathrm{Mb} / \mathrm{s}$. The bit-rate is an indicator for the amount of texture and motion occurring in a sequence. The Bus sequence with large, low-textured regions, and moderate motion has the smallest bit-rate of all test sequences $(12.6 \mathrm{Mb} / \mathrm{s})$. Shields and Stockholm are both moderately textured. The horizontal movement in the Shields sequence as well as the zooming operation of the camera in the Stockholm sequence result in a slightly higher motion activity than in the Bus sequence. The higher texturedness and motion activity for the Shields and Stockholm sequences lead to bit-rates of 18.8 and $25.6 \mathrm{Mb} / \mathrm{s}$, respectively. The natural environment in the Parkrun sequence contains strong texture patterns and a strong horizontal motion with various temporal occlusions. This leads to the highest bitrate in our test set $(50.8 \mathrm{Mb} / \mathrm{s})$.

\section{E. Dynamic Runtime Behavior of Macroblocks}

We have computed macroblock-based runtime measurements for the test sequences of Table I. Fig. 3 visualizes the MBs' decoding complexity for all four sequences. When comparing the complexity distributions of all four sequences, it is not surprising that the decoding complexity of individual MBs increases with higher bit-rates. The important point in Fig. 3, however, lies in the dynamic behavior of MBs. It can be clearly seen that cycle counts are very different among individual MBs. As is also shown in the figure, this observation can still be made when considering the classes of I, B, and $\mathrm{P}$-macroblocks alone. The pixel information that each $\mathrm{MB}$ encodes seems to have large influence on its runtime behavior.

Function profiling shows that all major function blocks of the decoder are strongly affected by this dynamic behavior of MBs. Analogously to before, the complexity for all function blocks increases with the bit-rate.

The strong impact of the video content on a MB's decoding complexity becomes obvious from the complexity profiles in Fig. 3 and the function profiling. Variations in the execution times of the decoding functions will result in a highly dynamic system when developing parallel decoders. Runtime prediction of a parallel decoding system is not straightforward in the light of this dynamic behavior. Moreover, multicore decoder simulation is complicated by dependencies that exist between individual MBs. These dependencies are described in the following.

\section{A Method for Simulating Video Processing ARCHITECTURES}

For a flexible description of the hardware as well as the video processing algorithm, we propose a high-level modeling approach. This approach is implemented by a tool that we refer to as the PAS. Fig. 4 illustrates the basic idea behind the PAS. To use the PAS, one has to give the following inputs. First, a description of the dependencies in a specific video algorithm is required. Second, the user provides an abstract description of the hardware as well as a partitioning of decoder functions onto this hardware. Third, the user presents macroblock profiling results of a specific stream to the PAS. The task of the PAS is then to simulate the runtime behavior of the described distributed decoder running on this virtual architecture. 


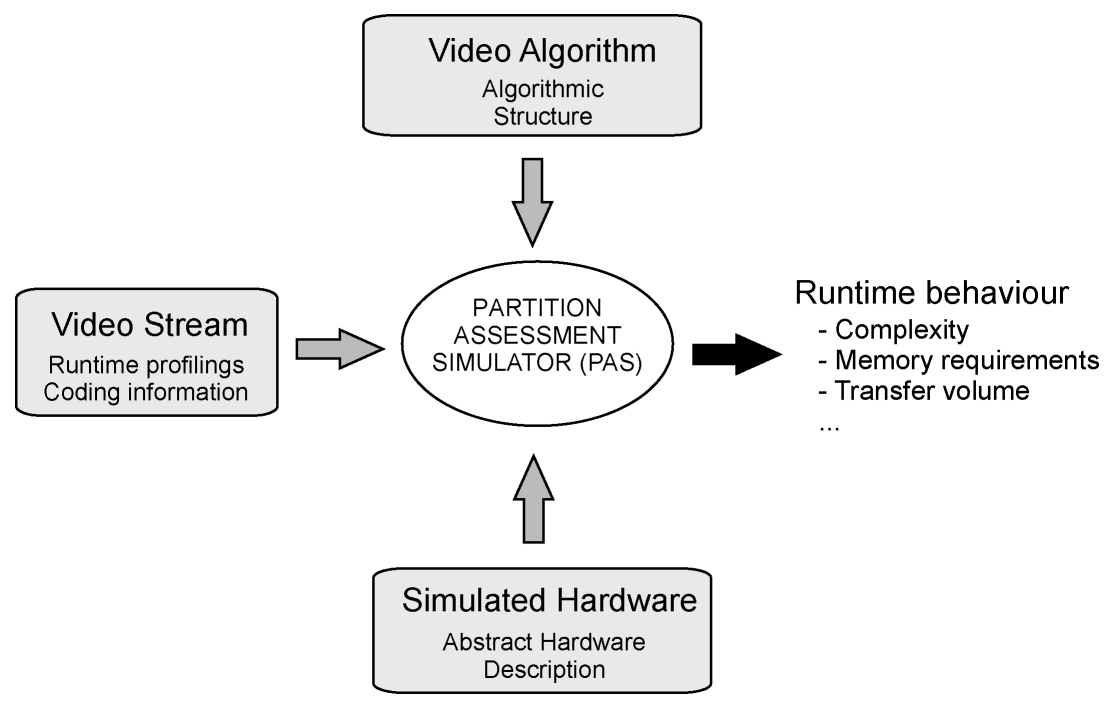

Fig. 4. Partition assessment simulator. Our simulator combines information about a video codec (e.g., H.264), a virtual multicore architecture, and a video input stream in order to estimate the runtime behavior of this specific video decoding architecture. Approximations of the decoder's performance (e.g., complexity, memory accesses, etc.) are accomplished without the need to fully implement the hardware or the decoder software.

Modeling the hardware and the software results in highflexibility when doing design space explorations. This includes the evaluation of unknown hardware configurations as well as software partitionings. For example, let us assume that we want to test a new multicore hardware system. In this case, the system designer just needs to provide an abstract description of the new hardware as well as an updated mapping of tasks to the given processing cores. These small modifications are already sufficient to estimate the runtime behavior of the VPA on the new hardware.

\section{A. Characteristics and Structure of VPAs}

Most video processing algorithms are highly data and complexity-intensive. For supporting an efficient and flexible implementation on state-of-the-art processing architectures, VPAs typically have the following characteristics.

1) They divide the input video into "architecture-friendly" processing packages (PPs) such as picture lines/columns, macroblocks, and frames. The processing tasks of a PP are clearly encapsulated by individual VPA functions (e.g., "filter MB 1").

2) The algorithmic dependencies strongly define the temporal order of the processing. Dependencies between the tasks of the same PP as well as between the tasks of different PPs occur.

3) They use efficient data load/store strategies for transferring the PPs between internal and external memories (e.g., block-based DMA transfers).

Note that these characteristics are independent of the video input signal (e.g., color space, bits per pixel, sampling rates, aspect ratio, interlace/progressive sampling, resolution) and the displaying (e.g., display rate). Our methodology exploits these characteristics for describing the parallel execution of VPAs in an efficient way. This makes the methodology well-suited for the modeling of VPAs.
Based on the similar structures of VPAs, we divide a video processing algorithm into $n$ tasks $T_{1}, T_{2}, \cdots, T_{n}$. The overall duration Dur $_{S C}$ for executing the VPA on a singlecore processor is computed by summing up the times spent on processing the individual tasks $T_{i}$ of all PPs

$$
\operatorname{Dur}_{S C}=\sum_{i=1}^{n} \operatorname{Dur}_{i}
$$

where $D u r_{i}$ denotes the duration of task $T_{i}$.

\section{B. Concept}

The proposed PAS uses profiling results that are obtained from single-core implementations. We have discussed the derivation of such single-core profiling results in the context of H.264 video decoding in Section III-B. For the following discussion, it is important to know that we have the duration $D_{i}$ for each task $T_{i}$ from single-core profiling. (An example of such a task is the entropy decoding of a specific MB.)

The idea behind our methodology is that a VPA running on a multicore architecture executes the same tasks as its single-core implementation. We make the assumption that a processor always requires the same number of computations for executing a specific task, no matter whether the processor is used in a single-core or multicore environment. Obviously, the usage of multiple processors enables parallel task execution. Our method aims to simulate this parallel execution of the tasks extracted from the single-core profiling. Consequently, the accuracy of the single-core profiling is of great importance for our simulation methodology. Additionally, we have to consider the major issues of parallel architecture executions such as the task assignment to parallel processing resources, concurrent task execution, inter-task communication and datadependencies between tasks.

For now, let us ignore dependencies that exist between tasks and assign each task to one of $p$ processors. We write $T_{i, j}$ to refer to task $i$ that is executed on processor $j$. Each processor 
$j$ executes $n_{j}$ tasks. In this simple model, it is easy to determine the runtime of the multicore decoder Dur $_{M C}$ by

$$
\operatorname{Dur}_{M C}=\max _{j=1 . . p}\left(\operatorname{Dur}_{M C, j}\right)
$$

where $D u r_{M C, j}$ is the duration processor $j$ needs to execute its assigned decoding tasks, which is computed by

$$
\operatorname{Dur}_{M C, j}=\sum_{i=1 . . n_{j}} \operatorname{Dur}_{i, j}
$$

The total duration $\operatorname{Dur}_{M C}$ of the VPA, therefore, depends on the processor which terminates last.

In a more realistic multicore VPA, the tasks will strongly depend on each other. These dependencies stem from the simple fact that one task usually needs the results of one or more other tasks as an input. The proposed PAS is capable to compute the overall runtime correctly with respect to such dependencies. The basic algorithm to accomplish this simulation is shown in Fig. 5. In the example of Fig. 5, we only consider a two-core system. However, the algorithm can easily be extended to handle an arbitrary number of processors. We go into more detail on the algorithm in the following.

The algorithm of Fig. 5 maintains three different kinds of sets. First, a set $\mathcal{T}_{i}$ represents all tasks that are executed on processor $i$. Second, we use sets $\mathcal{F}_{t}$ to keep track of all those tasks that are already completed at a specific instance of time $t$. Third, we introduce a dependency set $\mathcal{D}_{T}$ which is a list of all tasks that already need to be computed before we can start execution of the task $T$. We consider two types of dependencies, namely the intra and the inter-dependencies. Intra-dependencies (lines 6-10) occur between the tasks of the same processor. Inter-dependencies (lines 11-13) exist between the tasks of different processors.

The main loop of the algorithm (lines 17-45) is iterated until all tasks have been executed. For each processor, we determine the task $T$ that shall be executed next (line 23). This task can only be executed if its dependencies are already resolved. We, therefore, iterate through the dependency list of $T$ (lines 27-33) to check whether all depending tasks are already a member of the finished task list $\mathcal{F}_{t}$ at current time $t$. If this is the case, $T$ can be executed. We add $T$ to the list of finished tasks at time $t+\operatorname{Dur}_{T}$ and remove $T$ from the list of tasks that are still waiting for execution (lines 36-39). After the algorithm has left the main loop, the overall computation is determined from the value of the variable $t$ (line 45).

In the following sections, we will develop our methodology using a specific application, namely an H.264 video decoder. Based on this description, one can transfer this concept to other VPAs and architectures.

\section{Implementation}

In this section, we describe the user input for the PAS and the simulation process. Firstly, we define dependencies between the tasks. Secondly, we provide a virtual architecture description. The PAS resolves the task dependencies and computes the required decoder runtime.
1: // lists of tasks that are executed on two processors

2: $\mathcal{T}_{1}=\left\{T_{1,1}, T_{2,1}, \cdots, T_{n_{1}, 1}\right\}$

3: $\mathcal{T}_{2}=\left\{T_{1,2}, T_{2,2}, \cdots, T_{n_{2}, 2}\right\}$;

4: // list of tasks finished at times $0,1, \cdots, t_{\text {max }}$

5: $\mathcal{F}_{0}=\mathcal{F}_{1}=\cdots=\mathcal{F}_{t_{\text {max }}}=\emptyset$;

6: // specify intra-dependencies (just shown for processor 1)

7: $\mathcal{D}_{T_{1,1}}=\emptyset$;

8: $\mathcal{D}_{T_{2,1}}=\left\{T_{1,1}\right\}$;

9: :

10: $\mathcal{D}_{T_{n_{1}, 1}}=\left\{T_{1,1}, \cdots, T_{n_{1}-1,1}\right\}$;

11: // add an inter-dependency (e.g, task 3 of processor 1

12: // needs to wait for task 2 of processor 2)

13: $\mathcal{D}_{T_{3,1}}=\mathcal{D}_{T_{3,1}} \cup T_{2,2}$;

14: // the current time

15: $t=1$;

16: // loop while there are still tasks that need to be processed

17: while $\mathcal{T}_{1} \neq \emptyset \wedge \mathcal{T}_{2} \neq \emptyset$ do

$/ /$ tasks finished at $t-1$ are also finished at $t$

$\mathcal{F}_{t}=\mathcal{F}_{t} \cup \mathcal{F}_{t-1}$;

// for both processors

for $i=1$ to 2 do

$/ /$ access the first entry in the task list of processor $i$

$T=\mathcal{T}_{i}[1]$

$/ /$ check if all dependencies for $T$ are resolved

dependencies_resolved $=$ true;

// go through the dependency list of $T$

for $j=1$ to $\left|\mathcal{D}_{T}\right|$ do

// check if the task on which $T$ depends has

// already finished at time $t$

if $\mathcal{D}_{T}[j] \notin \mathcal{F}_{t}$ then

dependencies_resolved $=$ false; end if

end for

// in case that all dependencies are resolved

if dependencies_resolved $==$ true then

$/ /$ add $T$ to list of finished tasks at time $t+D u r_{T}$

$\mathcal{F}_{t+D u r_{T}}=\mathcal{F}_{t+D u r_{T}} \cup T$

// remove $T$ from the task list

$\mathcal{T}_{i}=\mathcal{T}_{i}-T$

end if

end for

$t=t+1$

43: end while

44: // computed execution time of the multi-core system

45: return $t$;

Fig. 5. Algorithm for simulating parallel task execution in a VPA. A detailed explanation is given in the text.

1) Defining Dependencies Between Tasks: The PAS maintains sets of dependencies similar to the ones in lines 7-10 of Fig. 5. At each time step $t$, it evaluates which dependencies are resolved. Fig. 6 illustrates when stalls occur during the execution of a parallel task.

The PAS delays the start/end of a task (i.e., it introduces stalls) if read/write-dependencies cannot be resolved. We can avoid stalls in the middle of a task by either breaking the task up into smaller tasks or by solving all read and writedependencies at the start and end, respectively. This results in read and write-dependencies only at the start and end of a task, respectively. In the PAS, we support the definition of these two types of dependencies in an efficient way. 


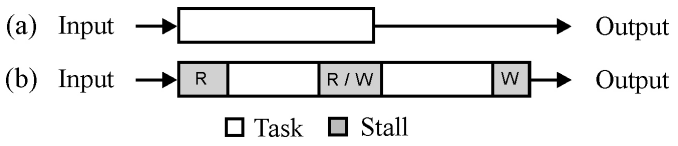

Fig. 6. Task execution on (a) single and (b) multicore architecture. (a) Task running on a single processor reads in and processes input data. The results are written to the output buffer. Since no parallel execution takes place, no stalls occur. In a multicore environment, read-dependencies on parallel executed tasks and write delays due to limited output buffers occur. This results in $\mathrm{read} / \mathrm{write}$ stalls at the beginning/end of a task. Between the start and the end of a task, read as well as write stalls occur.

Read-Dependencies: The dependencies between the tasks of an individual MB are typically determined by the decoding pipeline (Fig. 1). For example, the intra prediction task requires the execution of the entropy decoding task of the same MB. Fig. 7 shows how we define this dependency in the PAS. We generate dependencies between the intra and the entropy task of the same MB [i.e., task $T(i)$ and $T(j)$ have the same macroblock number]. We have extracted the type of each task automatically during the mapping to the parallelization level (Section III-B). The simulator accesses the type T(i).TYPE of a task $T(i)$ from the macroblock profiling.

More complex dependencies occur between the tasks of different MBs. For H.264, these dependencies are typically controlled by the input stream. For example, in H.264 the intra prediction mode determines which neighboring MBs are used for the prediction. This results in changing dependencies according to the intra prediction modes. For resolving these dependencies, we require additional information about the functionality of a decoding task (e.g., "Which intra prediction mode is used?").

The structuring of VPAs into PPs allows us to extract this information in an efficient way. The single-core software version of our decoder delivers all the information that is required for resolving stream-determined dependencies. We modify the decoder to print out the required coding information of a MB such as the intra prediction mode. We decode an individual stream with this decoder. In a similar way as for the macroblock profilings, a parsing script automatically maps the printed coding information to the individual tasks of a MB (e.g., "The intra prediction task of MB 1 uses $16 \times 16$ intracoding and the vertical mode."). The PAS stores this coding information for each MB's task. In addition to macroblockspecific coding information, we can provide global information about a specific stream such as frame width to the simulator. Note, that we describe the dependencies at the same level as the parallelization process. This has the advantage that no knowledge about lower layers and the dependencies there is required (e.g., dependencies between bits during the entropy coding). The labor-intensive work of describing the exact decoding functionality of each decoding task is thus avoided.

In the PAS, we define the read-dependencies based on the extracted tasks' properties such as coding mode and spatial/temporal position. The dependencies at parallelization level (i.e., for our H.264 decoder, the MB level) are typically well-known to the system designer. Using the MBs' properties, we can intuitively derive and express the read-dependencies

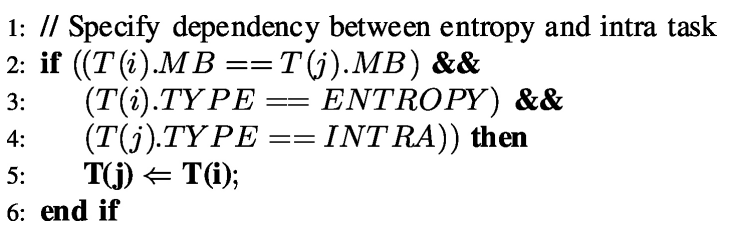

Fig. 7. Defining the dependencies between the entropy decoding and the intra prediction tasks of each macroblock. PAS evaluates at each time step $t$ if a task fits this rule and generates appropriate dependencies.

\section{1: // Assign entropy decoding tasks to Processor 1 \\ 2: if $(T(i) \cdot T Y P E==E N T R O P Y)$ then \\ 3: $\quad$ T(i).PROCESSOR = 1; \\ 4: end if}

Fig. 8. Assigning of tasks to a processor. All entropy decoding tasks are assigned to processor 1 .

between tasks from these dependencies. For specifying the execution flow of complex dependency rules, the PAS supports structures typically available in high-level programming languages such as for, while, and if structures.

Write-Dependencies: For determining write stalls between tasks, the PAS internally computes the amount of data which is exchanged between dependent tasks. The user defines communication first in, first outs (FIFOs) and connects the tasks to these FIFOs. For each task, we specify how much data is $\mathrm{read} / \mathrm{written}$ to a FIFO. We can extract this information from the decoder's source code (e.g., "the deblocking task reads $X$ Bytes"). The specification is done in a similar way as for the read-dependency rules. The PAS automatically computes the FIFO levels at each time step $t$ and delays a task if not sufficient memory for writing the task's results is available.

Furthermore, we define a transfer function for each FIFO. This function specifies the time a task requires for reading/writing to a FIFO. It allows us to model different memory properties such as latency and access time. The PAS delays the writing tasks automatically based on this transfer function.

2) Assigning VPA Tasks to Processors: The user defines a function which assigns each decoding task $T_{i}$ to a single processor $P_{j}$. The PAS uses the simplification that each task is completely executed on a single processor. For most application-tailored real-time systems, this is a small restriction. Dynamic scheduling of a task between multiple processors is rarely used since it requires an operating system for managing the task scheduling.

In Fig. 8, we assign all entropy decoding tasks to processor $P_{1}$. Similar to the read-dependencies, we can create more complicated assignments using the coding information of a MB. For example, for data-parallel partitioning techniques we can assign a task based on its MB's position. Additionally, we can exploit the structuring of VPAs into PPs and assign all tasks of a MB to the same processor.

At this point, the PAS knows the number of processors and the tasks executed on them. Using the dependencies, it determines the temporal order of each task during the decoder runtime. 

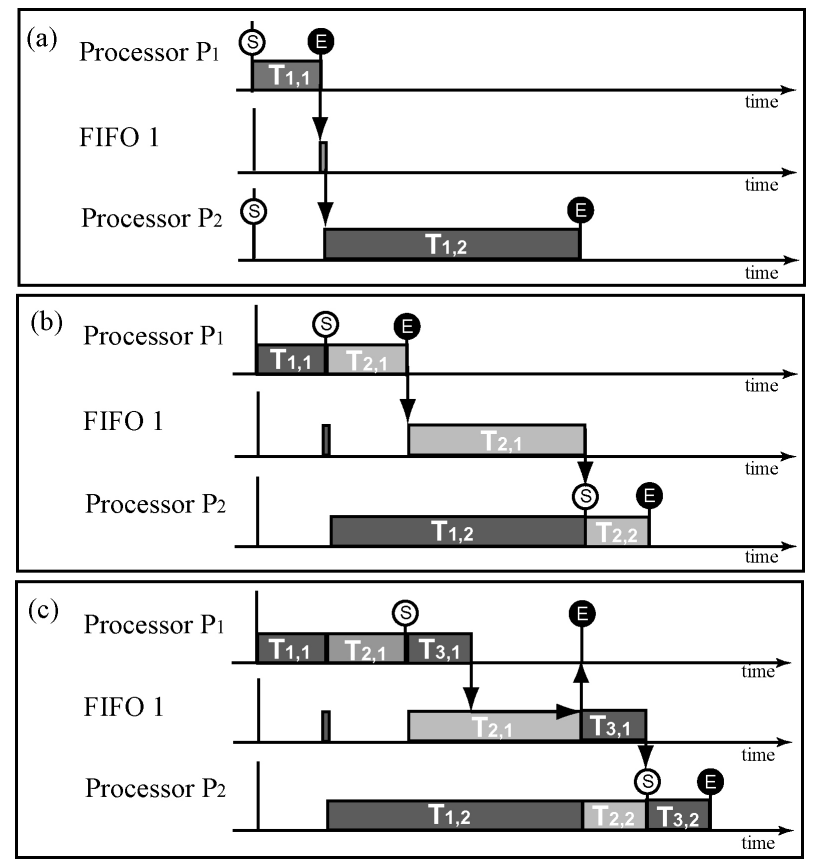

Fig. 9. Visualization of the internal simulation process in the PAS. Figure shows the execution states and buffer levels of two processors and one FIFO, respectively. Three macroblocks are processed on two individual processors, $P_{1}$ and $P_{2}$. After partially decoding each macroblock, $P_{1}$ writes the results to FIFO 1. For simplicity, the FIFO's maximum size is set to 1 macroblock. $P_{2}$ reads the macroblocks from this buffer and computes the remaining decoding tasks. Detailed explanation is given in the text.

\section{Multiprocessor Simulation}

Fig. 9 illustrates how the PAS works in more detail. The decoding process for three dependent MBs is shown. The decoding process of each $\mathrm{MB}$ has been divided into two tasks (i.e., function pipelining). $P_{1}$ performs the first decoding task $T_{*, 1}$ which includes the parsing and entropy decoding of each MB and writes the results to FIFO 1. In this example, the buffer size of FIFO 1 is limited to $1 \mathrm{MB} . P_{2}$ reads the results from FIFO 1 and applies the second decoding task $T_{*, 2}$.

The simulator sequentially processes one MB decoding task after another. For each decoding task, the PAS determines the dependencies on other tasks. At the simulation start, only the dependencies of task $T_{1,1}$ are completely resolved. The PAS evaluates when the assigned processor $P_{1}$ can start to execute $T_{1,1}$. In Fig. 9(a), this is indicated by the white marker (S). The processor's execution counter is increased by the task's duration. The black marker (E) indicates the end of a task. At this point $P_{1}$ has finished the task execution and written its results to the output buffer FIFO 1. At this stage, the PAS does not know when the data are removed by another task and marks the state of FIFO 1 as occupied.

In the next step, $P_{2}$ reads the macroblock data from the FIFO. When the PAS detects that $T_{1,2}$ can be started, it adapts the program counter of $P_{2}$ and frees the occupied memory in FIFO 1. A read stall due to data-dependencies occurs. $P_{2}$ cannot start its decoding operations simultaneously with $P_{1}$, but has to wait until the required data become available in the input buffer. The PAS uses the dependencies for detecting this read stall. The start of the task execution is delayed automatically.
In Fig. 9(b), the second MB is processed by $P_{1}$ and written to FIFO 1. After $P_{2}$ has finished its decoding operations for MB 1, it reads MB 2 and starts task $T_{2,2}$.

Fig. 9(c) visualizes a case where insufficient buffer is available and a write stall occurs. Since FIFO 1 has a maximum size of $1, P_{1}$ cannot write the results of decoding MB 3 immediately. It has to wait until $P_{2}$ has read MB 2 and freed the occupied memory in FIFO 1. A write stall occurs in this case. After finishing MB 2, $P_{2}$ reads MB 3 from FIFO 1 and decodes it.

The PAS uses the output-dependencies for considering limitations in the buffer communication (e.g., write stalls due to insufficient buffer sizes). The PAS delays the tasks automatically, if buffer scarcity occurs.

\section{E. Arbitrary Hardware Design}

In the previous sections, we have described the simulation method which the PAS is based on. Recall that in our method we assign the tasks extracted from a single-core profiling to multiple "virtual" processing units and estimate their runtime execution on such a parallel architecture. By using multiple single-core profilings from different processors, heterogeneous architectures based on these processors can be simulated. For example, if we want to build an architecture using two different types of processors we would provide two single-core runtime profilings to the PAS. Similar to the assignment of a task to a processor, we tell the PAS which of the two profilings contain the valid execution time of a task (e.g., "Use the runtime profiling of processor X for task Y"). The PAS extracts the task execution time from the specified profiling, maps this value to a unique time scale (e.g., seconds) and uses this value for further simulations. It should be noted that apart from instruction set profilings from programmable processors we can retrieve complexity information also from other traditional estimation techniques such as HDL simulations and mathematical complexity models. This allows us to simulate mixed architectures based on general (i.e., programmable) processing units and dedicated hardware.

\section{F. Calibration and Verification of the PAS}

Before the start of a design exploration, we have to calibrate the memory transfer times of the PAS to fit the transfer times of a typical processor architecture. In our case, we have used the CHILI-based environment described in Section III-A. We adjust the simulator configurations until a single-core PAS simulation achieves similar results as the decoder executed on the HW. Fig. 10 shows the comparison between the simulated and the measured runtime after the calibration. On average, the PAS shows differences of around 4\%. The highest differences are for the Parkrun sequence with $6 \%$.

For estimating the accuracy of our simulator, we partitioned the H.264 decoder into two parts. One part is doing the parsing and entropy decoding. The second part executes the reconstruction and the deblocking tasks. We measured the runtime of this decoder on a platform consisting of two CHILI processors with the same settings as described in Section III-A. Both processors share a $64 \mathrm{kB}$ on-chip SRAM for fast communication. We simulated the same decoder partitioning with 
(Runtime complexity)

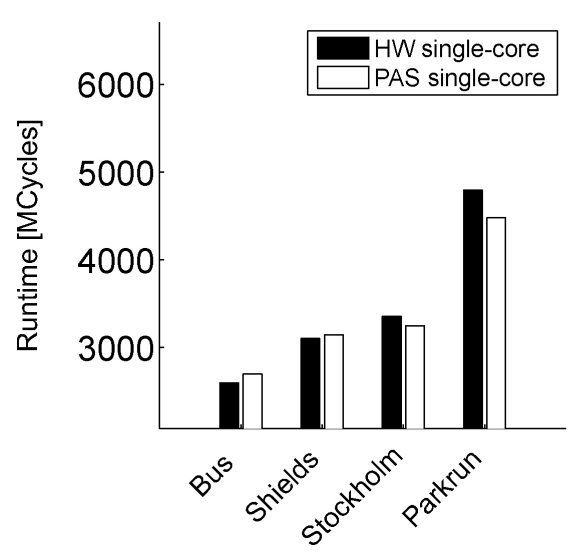

(Relative difference)

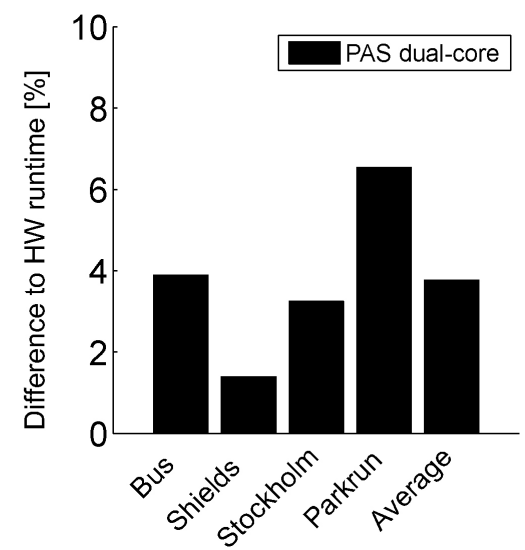

Fig. 10. Calibration of the PAS simulator: The PAS settings are calibrated according to the single-core profilings. The absolute runtime and the relative runtime difference between the HW single-core implementation and the PAS results after calibration are provided.

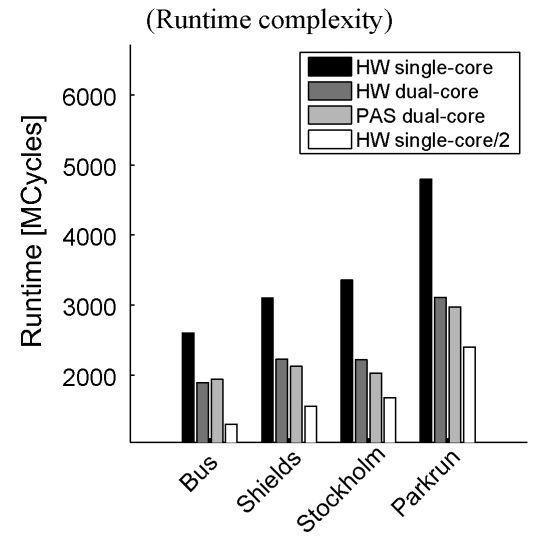

(a)

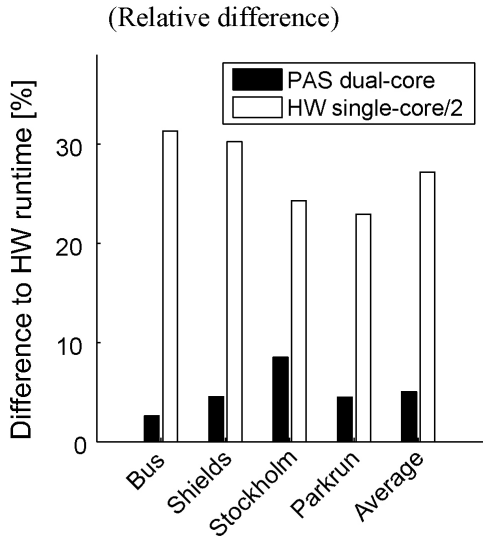

(b)

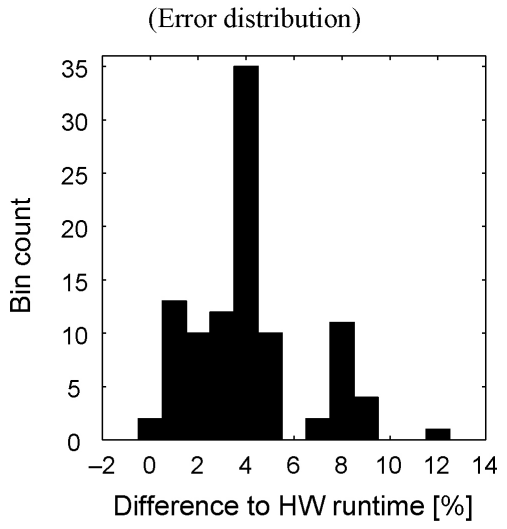

(c)

Fig. 11. Verification of the PAS simulator: (a) Absolute runtime difference between the single-core HW implementation, the dual-core HW implementation, the PAS estimation, and the simple estimation technique. Absolute runtime complexity measured on the HW is compared to the PAS-estimated runtime and a simple estimation technique dividing the single-core runtime by the number of cores. (b) Relative runtime difference between the dual-core HW implementation and the PAS estimation. (c) For each decoded frame in the test sequences, the end of the frame's decoding is measured on the HW and estimated with the PAS. The figure visualizes the distribution of the relative differences between the predictions and the measurements.

our calibrated PAS. Fig. 11 provides a comparison between the HW and the PAS-simulated system for the four test sequences. An average error of around 5\% indicates a good accuracy of the PAS [Fig. 11(b)]. Compared to a simple estimation technique where the runtime is divided by the number of processors, we achieve considerably better estimations. The Stockholm sequence shows the highest estimation error of $8 \%$. In addition to the absolute and relative runtime differences, we have analyzed the prediction accuracy of the PAS predictions during the decoding of a stream. We measured the end time of the decoding process for each frame using the HW and computed these values using the PAS. Fig. 11(c) visualizes the distribution of the relative differences between the PAS predictions and the HW measurements. The prediction error typically takes values between $3 \%$ and $5 \%$. However, in the worst case differences between the measurements and the predictions of up to $12 \%$ could be observed for our test sequences.

It should be noted that we apply the PAS at an early stage of the system design process. At this stage, little information about the complex interactions between the different components (e.g., processors, buses, memories) is available. This results in a high number of uncertainties when modeling an architecture. Hence, the PAS' prediction accuracy will depend on the system designer's knowledge and experience about the different hardware components.

\section{APPLiCATION OF THE PAS FOR H.264 DeCODING}

In this section, we demonstrate the application of the PAS for design explorations. The H.264 single-core decoder described in Section III is partitioned and mapped to a multicore system using our PAS. We aim to develop a parallel decoder for decoding $720 \mathrm{p}$ HD streams at 25 frames/s. Our decoder architecture is evaluated using the four test sequences described in Section III-D. CHILI processors form the processing units in our multicore decoder (i.e., CHILI profilings are used as input for the PAS). Each CHILI processor is run with a maximum core frequency of 

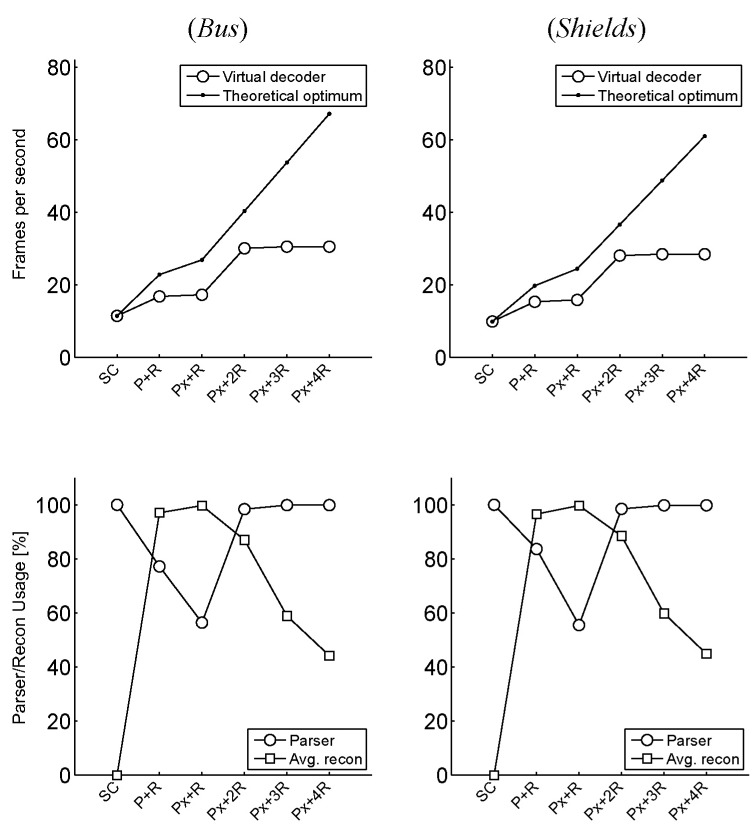

(a)

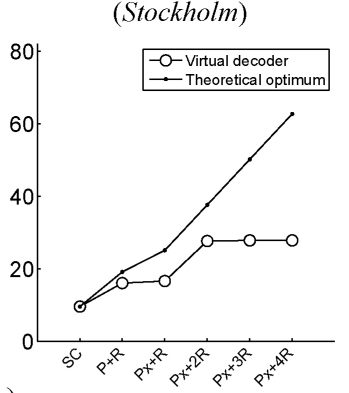

(a)

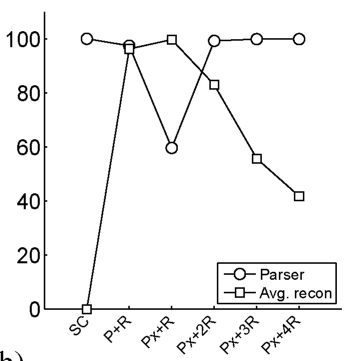

(b)

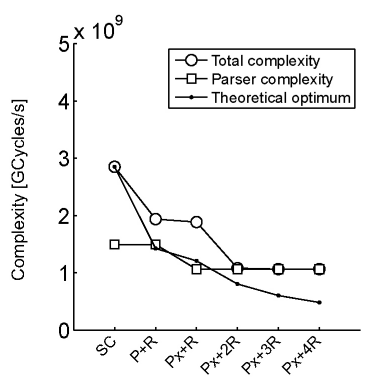

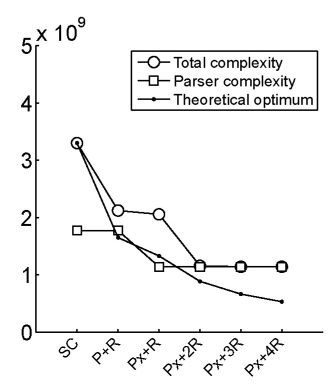

(c)
(Parkrun)
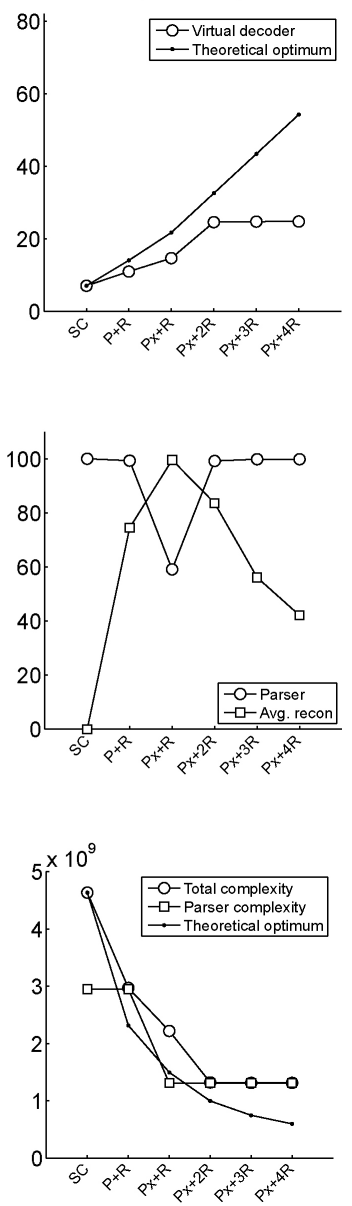

Fig. 12. Runtime of the simulated decoder partitioning approaches. (a) Frames per second achieved for various approaches are provided: the single-core decoder (SC), the functional split decoder into one parser and one reconstructor core ( $\mathrm{P}+\mathrm{R})$, and data-parallel partitionings of the reconstructor into up to four cores $(\mathrm{Px}+\mathrm{R}, \mathrm{Px}+2 \mathrm{R}, \mathrm{Px}+3 \mathrm{R}, \mathrm{Px}+4 \mathrm{R})$ using an extended CHILI core for parsing. (b) Usage of the cores running the parsing and the reconstruction tasks. The average usage for all available reconstruction tasks is shown. (c) Runtime complexity for the 4 sequences and the different approaches.

approximately $1.3 \mathrm{GHz}$. The PAS is calibrated as described in Section IV-F.

\section{A. Design Exploration}

1) Splitting 1-Functional Partitioning: Initially, our decoding system runs with a performance of 5-10 frames/s according to the PAS. In the first step of our decoder development, we move the computationally complex parsing and entropy decoding tasks onto one individual processing unit. We run the remaining functionality on a second core. The two cores are connected by a buffer that can hold one line of MBs (i.e., 80 MBs for 720 p). We apply the PAS to measure the performance of the two-core system. Fig. 12(a) shows the frame rates resulting from each decoder partitioning. For evaluating the results, the optimal frame rate increase is also visualized. In this context, optimal means that the performance of a system scales linearly with the number of cores in theory.

The functional splitting into parser and reconstructor $(\mathrm{P}+\mathrm{R})$ significantly reduces the runtime complexity of all four sequences. The sequences with higher bit-rates take the largest benefit from this functional splitting. For the Stockholm and the Parkrun sequences, the increases in frame rates are most closely to the optimal increase. Fig. 12(b) shows the usage for the parsing and the reconstruction cores. The reconstructor represents the bottleneck in our system, but also the parser is strongly occupied.

For evaluating how a faster parser influences such a system, we replaced the parsing core with an extended CHILI processor $(\mathrm{Px}+\mathrm{R})$. This processor provides hardware-acceleration for the bistream parsing and the entropy decoding (e.g., decoding of coefficients and exp-golomb coded syntax elements). Note that for simulating heterogeneous architectures with different types of processing units, the PAS uses multiple macroblock profilings as input.

A strong reduction in the parsing time can be observed. However, for the sequences with low bit-rates, the reconstructor stalls the system and the performance increases only moderately. For the Parkrun sequence, a faster parser improves the performance significantly.

The slow reconstructor and the limited buffering between the two cores result in write stalls at the parser side. We have three options for using the parser's computational resources more efficiently. Firstly, we can improve the recon- 
structor performance by moving it onto multiple processing units. Secondly, we can move additional tasks from the reconstructor (e.g., the filter strength calculation from the deblocking) to the parsing core. Thirdly, we could increase the buffer sizes between parser and reconstructor. All three options can easily be evaluated with the PAS. In the next section, we follow the first option, which is the most interesting approach in the context of multicore architecture design.

2) Splitting 2-Data-Parallel Reconstruction: Functional partitioning of the decoder reveals that the reconstructor is the bottleneck in our current system. By using a data-parallel decoding approach this part of the decoding process can be computed on multiple processing units. In this paper, we use a multicolumn approach for parallel macroblock processing [16]. Each frame is partitioned into vertical regions. Each of the regions is assigned to an individual processing unit. The reconstruction tasks for the MBs in this region are performed on this processing unit. This approach requires to consider the H.264 macroblock dependencies. Each processing unit can start with the decoding when all dependencies to neighboring regions have been resolved.

Fig. 12(a) shows the complexity reduction resulting from each additional core. Adding a second reconstructor $(\mathrm{Px}+2 \mathrm{R})$ strongly increases the frame rates for the all sequences according to our simulation results. For all sequences, the parsing core becomes the bottleneck in our decoder. Fig. 12(b) shows the core usage for the parsing core and the reconstruction core. Only around $80 \%$ of the reconstructors' execution time is effectively used for decoding. The parsing core runs at nearly $100 \%$ processor usage.

For three reconstructor cores $(\mathrm{Px}+3 \mathrm{R})$, the performance does not improve significantly. We can see that the stalling times caused by the slow parsing become even more obvious. The average usage of reconstructor cores indicates that the usage of each core is around $60 \%$. For 4 reconstruction cores $(\mathrm{Px}+4 \mathrm{R})$, approximately $40 \%$ of the reconstructors' execution time is used for decoding tasks.

Fig. 12(c) shows the complexity for the parsing and the complete decoding process in cycles/s. The parsing complexity currently requires approximately $1.25 \mathrm{G}$ cycles/s and determines the runtime of our decoding system.

\section{CONCLUSION}

In this paper, we have introduced a design methodology for flexible exploration of virtual multicore video processing architectures. We have implemented a simulator, the PAS, and verified the simulator's accuracy. As an application, we have analyzed the runtime behavior of different decoder partitionings. Using our simulator we have identified the critical performance bottlenecks of each multicore decoder and adapted our architecture accordingly. An H.264 decoder for 720 p HD resolution and running on multiple processing units has been developed. This decoder is able to decode H.264 video streams with resolutions up to $720 \mathrm{p}$ at 25 frames/s.

In future work, we are planning to analyze the proposed methodology for different video coding standards. We want to evaluate how information from the emerging area of reconfigurable video coding [17] can be incorporated into the PAS. Additionally, we want to model VPAs different from video decoding and simulate their behavior on parallel architectures.

\section{REFERENCES}

[1] C. H. Meenderinck, A. Azevedo, B. H. H. Juurlink, M. Alvarez, A. Ramirez, "Parallel scalability of video decoders," J. Sign. Process. Syst., vol. 57, no. 2, pp. 173-194, Aug. 2009.

[2] L. Fortnow and S. Homer, "A short history of computational complexity," Bull. Eur. Assoc. Theoretical Comput. Sci. Comput. Complexity Column, vol. 80, pp. 95-133, Jun. 2003.

[3] P. P. Puschner and C. Koza, "Calculating the maximum execution time of real-time programs," J. Real-Time Syst., vol. 1, no. 2, pp. 159-176, Sep. 1989.

[4] S. Malik, M. Martonosi, and Y.-T. S. Li, "Static timing analysis of embedded software," in Proc. 34th Assoc. Comput. Mach./IEEE Design Autom. Conf., Anaheim, CA, Jun. 1997, pp. 147-152.

[5] Y.-T. S. Li and S. Malik, "Performance analysis of embedded software using implicit path enumeration," in Proc. 32nd Assoc. Comput. Mach./IEEE Design Autom. Conf., Nov. 1995, pp. 456-461.

[6] S. L. Graham, P. B. Kessler, and M. K. McKusick, "gprof: A call graph execution profiler," in Proc. Special Interest Group Programming Languages Symp. Compiler Construction, vol. 17. Jun. 1982, pp. 120126.

[7] T. Ball and J. R. Larus, "Optimally profiling and tracing programs," Assoc. Comput. Mach. Trans. Programming Languages Syst., vol. 16, no. 14, pp. 1319-1360, Jul. 1994.

[8] B. Cmelik and D. Keppel, "Shade: A fast instruction-set simulator for execution profiling," in Proc. Assoc. Comput. Mach. Sigmetrics Conf. Measur. Model. Comput. Syst., May 1994, pp. 128-137.

[9] E. Witchel and M. Rosenblum, "Embra: Fast and flexible machine simulation," in Proc. Assoc. Comput. Mach. Sigmetrics Conf. Meas. Model. Comput. Syst., May 1996, pp. 68-79.

[10] M. Ravasi and M. Mattavelli, "High abstraction level complexity analysis and memory architecture simulations for multimedia algorithms," IEEE Trans. Circuits Syst. Video Technol., vol. 15, no. 5, pp. 673-684, May 2005.

[11] L. Nachtergaele, F. Catthoor, B. Kapoor, S. Janssens, and D. Moolenaar, "Low power storage exploration for H.263 video decoder," in Proc. $9^{\text {th }}$ Workshop Very Large Scale Integr. Sig. Process., San Francisco, CA, 1996, pp. 115-124.

[12] IEEE Standard for Verilog Hardware Description Language; IEEE Standard 1364-2001.

[13] W. O. Cesario, D. Lyonnard, G. Nicolescu, Y. Paviot, S. Yoo, A. A. Jerraya, L. Gauthier, and M. Diaz-Nava, "Multiprocessor SoC platforms: A component-based design approach," IEEE J. Des. Test Comput., vol. 19, no. 6, pp. 52-63, Nov.-Dec. 2002.

[14] S.-H. Wang, W.-H. Peng, Y. He, G.-Y. Lin, C.-Y. Lin, S.-C. Chang, C.-N. Wang, and T. Chiang, "A software-hardware co-implementation of MPEG-4 advanced video coding (AVC) decoder with block level pipelining," J. Very Large Scale Integr. Sig. Process. Syst., vol. 41, no. 1, pp. 93-110, Aug. 2005.

[15] F. Seitner, J. Meser, G. Schedelberger, A. Wasserbauer, M. Bleyer, M. Gelautz, M. Schutti, R. Schreier, P. Vaclavik, G. Krottendorfer, G. Truhlar, T. Bauernfeind, and P. Beham, "Design methodology for the SVENm multimedia engine," in Proc. Austrochip, Linz, Austria, Oct. 2008, p. 133.

[16] F. H. Seitner, M. Bleyer, R. M. Schreier, and M. Gelautz, "Evaluation of data-parallel splitting approaches for H.264 decoding," in Proc. 6th Int. Conf. Adv. Mobile Comput. Multimedia, Linz, Austria, 2008, pp. 40-49.

[17] E. S. Jang, J. Ohm, and M. Mattavelli, Whitepaper on Reconfigurable Video Coding (RVC), document N9586.doc, ISO/IEC JTC1/SC29/WG11, 2008. 


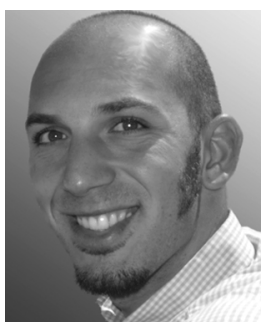

Florian H. Seitner (M'07) received the M.S. degree in computer science in 2006 from Vienna University of Technology, Vienna, Austria, where currently, he is working toward the Ph.D. degree.

Since September 2006, he has been employed as a Research Assistant at the Institute for Software Technology and Interactive Systems, Vienna University of Technology. His research interests include video coding, software and algorithm design, and image/video analysis.

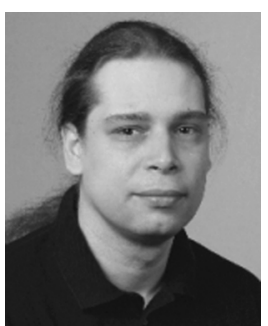

Michael Bleyer (M'09) received the M.S. degree in computer science from Vienna University of Technology, Vienna, Austria, in 2002, and the Ph.D. degree from the Institute of Software Technology and Interactive Systems, Vienna University of Technology, in 2006.

During his Ph.D. studies, he focused on the stereo matching problem, which is one of the most prominent research fields in computer vision. $\mathrm{He}$ is currently employed as a Postdoctoral Researcher at Vienna University of Technology. His research interests include stereo matching, optical flow computation, combinatorial optimization, and video coding.

For a paper on the sterero matching problem, he received the Best Paper Award from the International Society for the Photogrammetry and Remote Sensing Journal of Photogrammetry and Remote Sensing in 2004. His Ph.D. thesis, entitled "Segmentation-based Stereo and Motion with Occlusion," received the Ph.D. Thesis Award, given by the "Österreichischer Verband für Elektrotechnik."

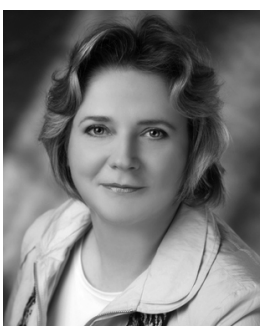

Margrit Gelautz (SM'08) received the M.S. degree in telematics and the Ph.D. degree in computer science from the Graz University of Technology, Graz, Austria, in 1992 and 1997, respectively.

She has over 15 years of experience in computer vision and related applications. During a Postdoctoral stay (1997-2000) at Stanford University, Stanford, CA, she worked on stereoscopic and interferometric radar image processing for remote sensing applications. Since 2005, she has been an Associate Professor at Vienna University of Technology, Vienna, Austria. Her current research interests include image and video analysis for multimedia applications.

Dr. Gelautz is the Women in Engineering Officer of the IEEE Austria Section.

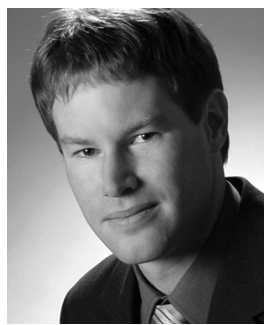

Ralf M. Beuschel was born in Friedrichhafen, Germany, in 1974. He received the M.Eng. degree in electrical engineering (systems engineering) from the University of Ulm, Ulm, Germany, in 2000.

From 2000 to 2006, he was a Research Fellow at the Department of Microelectronics, University of Ulm, where he does research in the field of low-delay video coding algorithms and real-time digital signal processing (DSP) implementations. Afterward, he was with the Institute of Software Technology and Interactive Systems, Vienna University of Technology, Vienna, Austria, for two years. In early 2009, he was hired at DSP-Weuffen, Amtzell, Germany, where he currently holds the position of a Senior System Designer for DSP video applications.

Mr. Beuschel is a Member of the German TV and Cinema Technology Society (Fernseh-und Kinotechnische Gesellschaft). He received the Best Master Thesis Award from the Verband Deutscher Elektrotechniker/Verein Deutscher Ingenieure." 